\title{
Weiterbildungsangebote für Hausarztmedizin in der Schweiz - eine Bestandsaufnahme
}

\author{
Djalali, S ; Tandjung, R
}

\begin{abstract}
Hausärztemangel wird viel diskutiert. Eine zukunftsgerichtete Lösung sind spezifische Weiterbildungsprogramme. Eine Studie analysiert die Charakteristika der aktuell bestehenden Angebote in der Schweiz.
\end{abstract}

Posted at the Zurich Open Repository and Archive, University of Zurich ZORA URL: https://doi.org/10.5167/uzh-95969

Journal Article

Published Version

Originally published at:

Djalali, S; Tandjung, R (2014). Weiterbildungsangebote für Hausarztmedizin in der Schweiz - eine Bestandsaufnahme. PrimaryCare, 14(8):139. 


\section{Weiterbildungsangebote für Hausarztmedizin in der Schweiz - eine Bestandsaufnahme}

\begin{abstract}
Über den Hausärztemangel wird viel diskutiert. Eine zukunftsgerichtete Lösung sind spezifische Weiterbildungsprogramme. Eine Studie analysiert die Charakteristika der aktuell bestehenden Angebote in der Schweiz.

Viele Fertigkeiten für die Hausarztmedizin können nur in Fachkliniken erlernt werden. Das Problem: Selten werden kurzzeitige Anstellungen (etwa sechs Monate) angeboten, die einem angehenden Hausarzt einerseits eine solide Basis in einem Spezialfach geben, andererseits so kurz sind, dass mehrere solcher Rotationen während der Weiterbildungszeit absolviert werden können. Ein angehender Hausarzt ist darum zwangsläufig entweder mit einer verlängerten Weiterbildungszeit oder mit einer Einschränkung seiner medizinischen Bandbreite konfrontiert.
\end{abstract}

\section{Curricula als Lösung}

Vielerorts wurden die Herausforderungen erkannt und als Lösung Praxisassistenzen und/oder spezifische hausärztliche Curricula eingeführt, die kürzere Anstellungen in spezifischen Rotationen ermöglichen. In der Regel werden sie durch Subventionen finanziert. In einer Übersichtsarbeit [1] wurden die aktuell zur Verfügung stehenden Weiterbildungsangebote für Praxisassistenzen und hausärztliche Curricula, die in den verschiedenen Kantonen existieren, systematisch beschrieben und verglichen.

\section{Umfangreiche Recherche}

Als Datengrundlage diente eine umfangreiche Internetrecherche auf allen Webauftritten der kantonalen Gesundheitsdirektionen, der kantonalen (Haus-)Ärztegesellschaften, der universitären Institute für Hausarztmedizin sowie der grossen Spitäler. Die Ergebnisse wurden mit der bereits bestehenden Übersicht der Stiftung zur Förderung der Weiterbildung in der Hausarztmedizin (WHM) abgeglichen. Zudem wurden die Kontaktpersonen der jeweiligen Programme für weiterführende Informationen bzw. Bestätigung der Informationen per E-Mail oder Telefon kontaktiert.

\section{Mehr Praxisassistenzen, wenig Curricula}

Die Ergebnisse zeigen, dass hausarztspezifische Weiterbildungsangebote in der Schweiz zunehmend ausgebaut werden. Fast überall im Land ist heute der Anschluss an eine Praxisassistenz möglich. Hausarztspezifische Curricula, die über eine Praxisassistenz hinausgehen sind jedoch noch selten (Angebote in 10 Kantonen). Eine Hürde für junge Mediziner könnte die Niederlassungspflicht sein.

\section{Niederlassungspflicht}

Zu Beginn der kantonalen Praxisassistenzprogramme war es üblich, dass die Teilnehmer sich verpflichteten, innerhalb einiger Jahre nach Abschluss des Programms als Grundversorger im Kanton zu praktizieren. Mittlerweile wird bei Praxisassistenzen ausser in Basel-Stadt darauf verzichtet. Anders sieht es bei den Curricula aus, dort bleibt eine Niederlassungspflicht von 5-7 Jahren die Regel. Eine Variante ist die Rückzahlungspflicht:

Bei Nicht-Erreichen eines Grundversorgerfacharzttitels innerhalb von acht Jahren nach Programmabschluss ist der Teilnehmer verpflichtet, die Hälfte der Unterstützungsbeiträge zurückzuzahlen.

\section{Fehlende Evaluation}

Auffallend ist, dass die Evaluation der einzelnen Programme heute noch nicht systematisch und nicht flächendeckend erfolgt. Wenn evaluiert wird, dann auf Basis von Meinungserhebungen des Praxisassistenten, des Lehrpraktikers und des medizinischen Praxispersonals, wobei Lerninhalte bezüglich des hausärztlichen Wissens und der hausärztlichen Fertigkeiten (z. B. Laboruntersuchungen, Umgang mit Röntgen) geprüft werden. Eine zusätzliche Überprüfung der Weiterbildungsqualität etwa durch Bewertung der durch den Lehrpraktiker gebotenen Betreuung, wird nur in einem Praxisassistenzprogramm beschrieben. Auch publizierte Daten sind rar, was eine Beurteilung der Qualität und Effektivität erschwert.

\section{Fazit}

Eine schweizweite Vereinheitlichung der Koordination und der Finanzierungsschlüssel der Weiterbildungsprogramme dürfte es in Zukunft erleichtern, von den Niederlassungspflichten im Kanton Abstand zu nehmen. Die Programme sollten regelmässig evaluiert und die Daten über Qualität und Wirkungsnachweis publiziert werden.

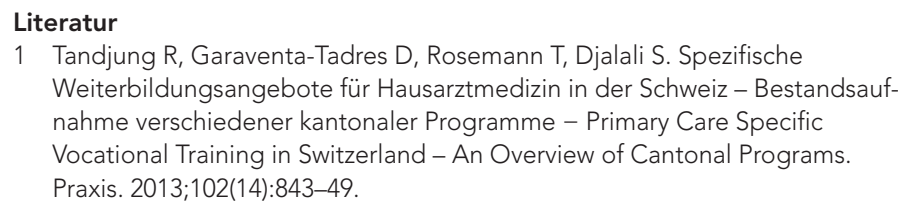

Dr. med. Sima Djalali, Wissenschaftliche Mitarbeiterin

Institut für Hausarztmedizin, Universität Zürich, Pestalozzistrasse 24

8091 Zürich, sima.djalali[at]usz.ch, www.hausarztmedizin.uzh.ch

\section{PrimaryResearch - das Fenster zur Forschung}

In dieser Artikelserie stellen wir Forschungsarbeiten aus dem Institut für Hausarztmedizin der Universität Zürich (IHAMZ) vor. Die Originalarbeiten sind entweder «open access» zugänglich oder beim jeweiligen Autor auf Anfrage erhältlich. Die Ergebnisse geben einen spannenden Einblick in die täglichen Herausforderungen, aber auch die Leistung der Hausarztmedizin. An dieser Stelle ein herzlicher Dank an alle Kolleginnen und Kollegen, die sich an den Projekten beteiligen und die hier präsentierten Ergebnisse erst ermöglicht haben!

Universität

Zürich $^{\text {vz }}$

Institut für Hausarztmedizin 